\title{
Acquisition and Transfer of English as a Second Language through the Constructional Response Matching-to-Sample Procedure for Students with Developmental Disabilities*
}

\author{
Mikimasa Omori ${ }^{1}$, Hiroshi Sugasawara ${ }^{2}$, Jun-ichi Yamamoto ${ }^{3}$ \\ ${ }^{1}$ Department of Psychology, Graduate school of Human Relation, Keio University, Japan; \\ ${ }^{2}$ College of Human Science, Tokiwa University, Japan; \\ ${ }^{3}$ Department of Psychology, Faculty of Letter, Keio University, Japan. \\ Email: mo carzy0219@a5.keio.jp \\ Received May 12 $2^{\text {th }}, 2011$; revised July $13^{\text {th }}, 2011$; accepted August $15^{\text {th }}, 2011$.
}

\begin{abstract}
Japanese students, who study English as second language often have the difficulty in learning English. Students with Autism Spectrum Disorders (ASD) have special difficulty with English spelling. Previous researches suggest that equivalence-based training, such as the matching-to-sample (MTS) procedure and the constructional response matching-to-sample (CRMTS) procedure were effective for the acquisition of spelling. The present study examined the controlling variables for the acquisition of English spelling skills by 4 Japanese students with ASD and compared the effects of MTS and CRMTS procedures on transfer to spelling. The results showed that the MTS and CRMTS procedures were equally effective for shorter letter words. But the students showed better results on acquisition and transfer to spelling for longer letter words after CRMTS procedure. The results are discussed in terms of the effect of the CRMTS procedure on the acquisition and transfer of spelling words.
\end{abstract}

Keywords: Matching-to-Sample, Constructional-Response Matching-to-Sample, English as a Second Language, Developmental Disabilities, Spelling

\section{Introduction}

When acquiring reading and writing, we construct the stimulus relationship between three types of stimuli, pictures, written letters and sounds (Sidman, 2000; Sugasawara \& Yamamoto, 2007). However, students with developmental disabilities have difficulties in acquiring these types of stimulus relationship in reading and writing. In Japan, the English as a second language (ESL) school curriculum begins in the first year of junior high school when students are usually 12 or 13 years old. They will spend approximately six years studying English. According to the official curriculum, four basic skills of English are taught: listening, speaking, reading, and writing (Ministry of Education, Culture, Sports, Science and Technology, 2002).

In learning writing skill, motor coordination is required. Students with developmental disabilities such as autism spectrum disorder (ASD) often have difficulties in motor development including deficits in motor planning, motor coordination and so on (Hauck \& Dewey, 2001). They show some difficulties in holding and moving a pencil (Erhardt, 1988), and in writing down words or sentences that are dictated (Ohba, 1996).

Because of the problem of poor motor development, training without hand writing by using a computer is an effective and efficient way of teaching writing to students with developmental disabilities (Vedora \& Stromer, 2007; Yamamoto \& Miya, 1999; Yamamoto \& Shimizu, 2001). In previous research, two teaching strategies have been proposed to instruct these students in writing: the arbitrary matching-to-sample (MTS) procedure (Stromer, Mackay, Howell, McVay, \& Flusser, 1996) and the constructional response matching-to-sample (CRMTS)

${ }^{*}$ Global Center of Excellence (GCOE) program, Keio University Japanese Society for the Promotion of Science (JSPS). procedure (de Rose, de Souza, \& Hanna, 1996; Vedora \& Stromer, 2007). In a typical MTS paradigm, the student is required to select the one comparison stimulus word that best corresponds to the sample stimulus. For example, a picture of cup was presented as a sample stimulus, and the student was required to choose a corresponding word, "cup," from comparison word stimuli; cup, cap, and dog (Stromer et al., 1996). After the participant made a correct response, he or she received a token as a reinforcer. On the other hand, in a typical CRMTS paradigm, a picture is used as the sample stimulus and the letters or characters comprising the target word are the comparison stimuli. For example, in the study of Vedora and Stromer (2007), a picture of bread was presented as a sample stimulus, and the student was required to touch letters $b, r, e, a$, and $d$ sequentially from the collection of letters presented on the computer in order to construct a word "bread." After the participant made a correct response, they received a token as a reinforcer.

Computer-based training that involves choosing or constructing a response, such as MTS and CRMTS, often results in not only the acquisition of stimulus relationships, but also transfer to hand writing on paper. For example, Stromer, Mackay, Howell, McVay, and Flusser (1996) showed that transfer to spelling on paper occurred as a consequent effect of the MTS procedure. In their study, students were required to choose a comparison printed word in the presence of a sample picture, and then write the name of the picture. In addition, Vedora and Stromer (2007) showed that transfer to tabletop spelling occurred as an effect of the constructional response. They showed that students with developmental disabilities acquired and improved their reading and writing skills for words through CRMTS procedure. In their study, students were required to construct letters for a word sequentially on the computer, and then write the name of the picture and dictated word. In the 
previous studies, the participants acquired writing skills for their first languages. Few studies, however, suggested that this type of training is as effective for ESL students. In our procedure, when a picture was presented on the computer as a sample stimulus, the student was required to write down the name of picture in English on paper. The student was also required to listen to the Japanese sounds and translate the sound into English written words by writing them down on paper. What is more, although the MTS and CRMTS procedures are have been shown to be effective for children with developmental disabilities in acquiring writing skill (Stromer, et al., 1996; Stromer et al., 1998; Vedora \& Stromer, 2007; Sugasawara \& Yamamoto, 2007), no study has indicated which procedure is more effective for learning and transfer to hand-writing.

English words consist of strings of letters. For example, the word "dog" consists of three letters, d, o, and g. Previous studies reported that 3, 4, and 5 letter words could be acquired and transferred to hand-writing through the MTS and the CRMTS procedures (Stromer et al., 1996; Stromer et al., 1998; Vedora $\&$ Stromer, 2007). However, 3 to 5 letter-words were too short to compare the effects of the two types of training. Further, no studies have indicated how word- length affects acquisition and transfer to hand-writing through the MTS and the CRMTS procedures. In order to compare the effects of the two types of training, we prepared $3,4,5,6,7$, and 8 letter words as our target stimuli.

When we use MTS and CRMTS procedure, we often use generalized reinforcer such as token (Stromer, et al., 1996; Stromer et al., 1998; Vedora \& Stromer, 2007). However, when the participant received a differential feedback such as reinforcing stimuli, reinforcing stimuli can be used as a reinforcer. Dube and McIlvane (1995) found that reinforcing stimuli can construct same stimulus relationship with sample and comparison stimuli. They also indicated that reinforcing stimuli would be included in same stimulus class of sample and comparison stimuli (Dube \& McIlvane, 1995). In addition, Layng, Twyman, and Stikeleather (2004) reported that by combining two separate stimuli underlying two different behaviors into a single presentation, a new behavioral blend would be produced via a contingent consequence. The differenttial feedback procedure provides a specific reinforcing stimulus after a particular response is selected in the MTS procedure (Yamamoto \& Shimizu, 2001).

Sugasawara and Yamamoto (2007) showed that two students with developmental disabilities could acquire reading skills through the CRMTS with the differential feedback procedure even though they were not given reading training for each character. For example, a picture of a cat was the sample stimulus and the Japanese phonograms (Hiragana) characters “权 $(/ \mathrm{ne} /)$ ” and “こ(/ko/)" were the comparison stimuli in the CRMTS procedure. When the student chose the character ' 12 , as a comparison stimulus, the sound of "ne" followed as the differential feedback. When the student subsequently chose the character "こ" as a comparison stimulus, the sound of "ko" followed as the differential feedback. When the student constructed the correct word “㱛こ," a fanfare and a circle was presented as a reinforcer and the sound of "ne-ko" was presented as the differential feedback. This procedure could facilitate the acquisition of the specific relationship between the sample stimulus, the selected comparison stimulus, and the reinforcing stimulus (Dube \& McIlvane, 1995; Sidman, 2000; Yamamoto \& Shimizu, 2001). Although these studies showed that the students could acquire reading skill through MTS or CRMTS with the differential feedback procedure, the results were limited to the students' first language. Therefore, in the present study, we also examined the acquisition of English reading skill through both types of training with the differential feedback procedure.

In the present study, we examined three things. First, we compared the effects of the MTS and CRMTS procedures on ESL students with autism spectrum disorders. We examined which procedure was more effective to facilitate the acquisition and transfer of stimulus relationships by comparing the percentage of correct responses to English writing tests after each training procedure. Second, we examined how word-length affects acquisition and transfer to hand writing through the MTS and CRMTS procedures. While previous research (Stromer et al., 1996; Stromer et al., 1998; Vedora \& Stromer, 2007) used 3, 4 , or 5 letter-words for the target stimuli, we prepared three 3 to 8 letter-words to examine the difference. Third, we evaluated English reading skills of ESL students to examine whether English spoken sound stimuli presented as differential feedback could be used to construct stimulus relationships with pictures and written letters, and whether the acquisition of English reading skill was different with the MTS and CRMTS procedures.

\section{Methods}

\section{Participants}

Four male students diagnosed with Autism spectrum disorders (ASD) participated in the present study. They were recruited from Tokyo region via letter sent to parents. Informed consent was provided to both students and parents. All of them agreed to participate in the present study. Their profiles are shown in Table 1. Assumed names are used to identify all of the students.

Tomo was 15years old, Ryo was 17 years old, Atsushi was 13years old, and Sho was 15years old. Their mean age was 15 years old. All of the students were enrolled in mainstream schools at the time of the present study. They didn't show any difficulty in Japanese writing, speaking, and listening. We evaluated their English skills. The students were required to write English letters from " $A$ " to " $Z$ " in upper and lower-case and required to read English letters from " $A$ " to " $Z$ " in upper and lower-case. All of them could write down English letters and could pronounce each letter clearly. They could also discriminate upper- and lower-case English letters. Their mean full Intelligence Quotient (IQ) was 82.00 in Wechsler Intelligence Scale for Children-Third Edition (WISC-III) (Japanese edition; Wechsler, 1998), with a range from 78 to 84 , mean verbal IQ

Table 1.

Profiles and WISC-III scores of students.

\begin{tabular}{cccccc}
\hline Students & Diagnosis & age & FIQ & VIQ & PIQ \\
\hline Tomo & Autism & 15 & 84 & 89 & 82 \\
Ryo & Asperger's syndrome & 17 & 82 & 83 & 82 \\
Atsushi & Autism & 13 & 78 & 63 & 99 \\
Sho & Autism & 15 & 84 & 74 & 99 \\
Mean & & 15.00 & 82.00 & 77.25 & 90.50 \\
\hline
\end{tabular}

Note. IQ scores were measured by using WISC-III. All students are identified by assumed names. 
was 77.25 , with a range from 63 to 89 , and mean performance IQ was 90.50 , with a range from 82 to 99 . Even though their mean performance IQ was below average, all showed no difficulty using pencils or typing on keyboards.

\section{Materials \& Setting}

A laptop computer (Toshiba, Dynabook TX/470LS) was used to present stimuli and for trainings. We prepared the two training procedures, MTS and CRMTS, on the computer which was programmed using Adobe Macromedia Director ${ }^{\circledR}$. The sample stimuli were $200 \times 150$ pixels in size and were constructed using Adobe Photoshop CS3.

The teaching programs were conducted in a laboratory. In both procedures, one block consisted of eight trials. The computer recorded the students' responses during the training.

In both procedures, a picture was presented as a sample stimulus for each trial. Both procedures presented the sound of the word as the differential feedback when the students made a correct response. For example, a picture of bat was the sample stimulus and the written word "bat" was the correct comparison stimulus in both procedures.

In MTS procedure, the student was asked to choose the word "bat" from among 4 written comparison stimuli. In the CRMTS procedure, the student was asked to type letters $b, a$, and $t$ sequentially on the keyboard in order to construct a word "bat." When students chose or typed correctly, the sound /bæt/ was presented as the differential feedback. On each trial, a picture was presented as the sample stimulus on the computer. Then the comparison stimuli were presented, and the student was required to choose or type the word corresponding to the sample stimulus. If a correct response was made, a fanfare and a circle were presented as a reinforcer, and the sound of the word was presented as the differential feedback. If a correct response was not made, the same trial was repeated until the student could make a correct response.

Pencils and papers were provided for the writing test before and after trainings. Sample stimuli were Picture, English written words presented on the computer and Japanese spoken sound, spoken by the experimenter. They were required to write down English words on paper. Voice recorder was prepared for the reading test which the participant took before and after trainings. Sample stimuli were English written words presented on the computer. They were required to read English words toward voice recorders so that their reading response could be recorded.

\section{Stimulus Sets}

Seventy English noun words were prepared and categorized according to word length. For example, "dog" was categorized as a three-letter word, and "lion" was categorized as a fourletter word. A set consisted of four words from the same wordlength category.

\section{Experimental Designs}

A multiple pre-post design (Sugasawara \& Yamamoto, 2007; Sugasawara \& Yamamoto, 2009) was used for the present study. In order to compare the training effect of both the MTS and CRMTS procedures, a training session was implemented between pre- and post tests. First, the students took two types of pre-test within a specific word-length category. Then, their training started with either the MTS procedure or the CRMTS procedure. When students finished either of the training sessions, four types of post test were taken. After that, the other training within the same word-length category started and lasted until the post tests were finished. The numbers of training blocks were equated between the two training procedures within the same word-length category. Students then began a new word-length category. To diminish the possibility that students would learn the correct responses due to the order of training, we quasi-randomized the order of training and changed the stimulus set for each student.

\section{Procedures}

We conducted a pre-assessment, pre-test, training, and post test. If the participant made less than $100 \%$ correct responses for two consecutive blocks in training, we trained him on the same stimulus set again. When the participant passed the criterion, we gave him four types of post test. After that, the other training procedure was conducted.

\section{Pre-assessment}

First, seventy pictures were presented on the computer oneby-one. The students were instructed to write down the name of the pictures presented on the computer and name them verbally in English. We selected unknown words as stimuli. We defined unknown words as the words that students were unable to write down when shown pictures and were unable to name verbally in English before training.

There were six word-length categories, from three to eight letter words. Eight unknown words which were different in each student for each word-length category were selected by this assessment procedure. The eight selected words were divided into two sets of four words, with each set having words from the same word-length category.

\section{Pre-test}

The students received two types of pre-test. Both tests consisted of eight trials. Sample stimuli were shown on the computer in both tests. First, in the pre-test for English name writing, students wrote by hand on paper the name of the picture shown on the computer. In the other test, the pre-test for English written word reading, students were required to read the English written word aloud presented on the computer. After these tests, the training session started.

\section{Training}

During the training session, the students began with either the MTS procedure or the CRMTS procedure. We quasi-randomized the order of training and changed the stimulus set for each student in order to diminish the possibility of learning the correct response by order. Figure 1 shows the paradigm of the two training procedures, MTS (top) and CRMTS (bottom). In both procedures, when students made the correct response, a fanfare and a circle was presented as a reinforcer and the sound of the word was immediately presented once as the differential feedback. In both training procedures, four words from the same word-length category were chosen as one set. One block consisted of only either the MTS training or CRMTS training. In training, one block consisted of eight trials and within a block, each sample stimulus presented twice. In both training procedures, the first two blocks were prompt blocks in which the experimenter showed the participant how to use the programs and instructed them to the correct responses. In all trials, when a correct response was made, a circle and fanfare sound was presented as a reinforcer and then the sound of the word as the differential feedback was presented as well. When an incorrect response was made, the display did not change, but an incorrect response was recorded. Then the trial was repeated 

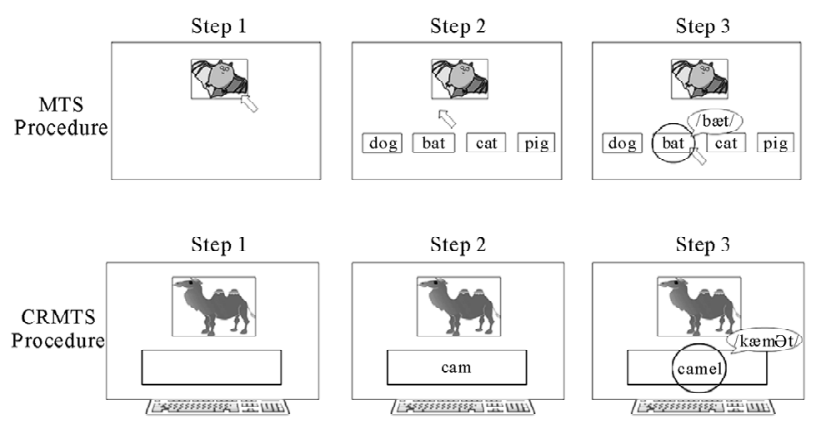

Figure 1.

Sequence of events in a trial for MTS and CRMTS training procedures. Students started with either the MTS (top) or the CRMTS procedure (bottom). Both procedures started from Step 1 through Step 3. In both procedures, when students made the correct response, a reinforcer, a circle and fanfare sound, was presented and the sound of the word was immediately presented once as the differential feedback (Step 3).

and another response was required. Each training procedures lasted until students met the criterion of scoring $100 \%$ in two consecutive blocks.

Figure 2 shows the relationship of writing and reading skills. In Figure 2, black line represents the trained relationship between picture and English written word and gray line represents the English spoken sound as the differential feedback. Black dotted lines show the transfer to writing and reading responses from trained relationship on the computer and differential feedback

\section{Matching-to-Sample (MTS) Training}

In the MTS training procedure, a picture was first presented in the upper part of the display as a sample stimulus.

When the participant clicked the picture, four comparison word stimuli appeared in the lower part of the display. The four comparison stimuli corresponded to the four picture stimuli in the set. The placement of the comparison stimuli was quasirandomized on each trial. The participant was required to choose the word which best described the picture within 2 seconds after clicking the picture. If the participant couldn't respond within 2 seconds, a new picture stimulus was presented on the computer for a new trial. When the participant made mistakes twice in a trial, the experimenter pointed the name of the picture presented on the computer to lead a correct response.

\section{Constructional Response Matching-to-Sample (CRMTS) Training}

The other training procedure was the CRMTS procedure. First, a picture stimulus was presented in the middle part of the display, with a frame below. When the sample stimulus was presented, the participant was given 10 seconds to type the name of the picture. If the participant couldn't type the word in 10 seconds, a new picture stimulus was presented on the computer for a new trial. When the participant made mistakes twice in a trial, the experimenter told him the name of the picture by saying each letter in order to make a correct response.

\section{Post Test}

In the post test phase, we examined whether students had acquired English writing and reading skills through the two training procedures. After two types of training, the students received four types of post test. Two tests were conducted to evaluate their writing accuracy.

Writing test 1: picture to English written word (Writing 1:

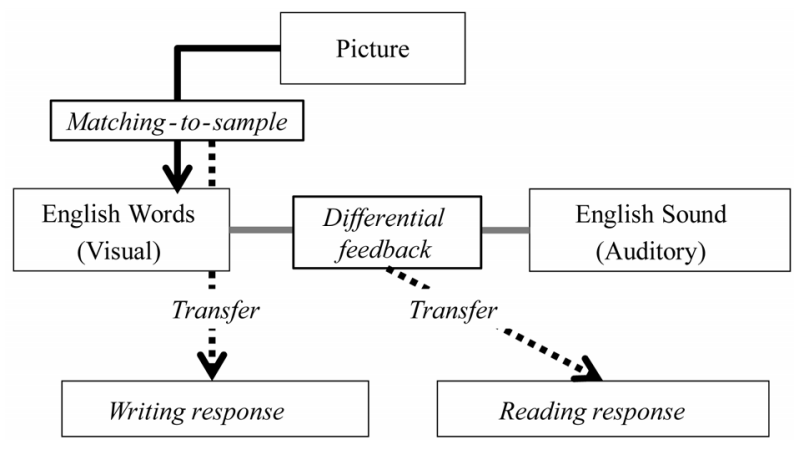

Figure 2.

The relationships of writing and reading skills. Black line represents the trained relationship between picture and English written word and gray line represents the English spoken sound given to the chosen word as the differential feedback. Black dot lines show the transfer to writing and reading skills from trained relationship on the computer and differential feedback.

picture to English). The students wrote by hand the name of the picture shown on the computer.

Writing test 2: Japanese spoken sound to English written word (Writing 2: Japanese to English). This test was a new test because students weren't trained the relationship between Japanese spoken sound and English written word. In this post test, the spoken sound of a Japanese word was presented as a sample stimulus and students were required to listen to it and translate it into English by hand-writing.

Reading test 1: English written word to English spoken sound (Reading 1: English to English). The students were required to read the English written word shown on the computer.

Reading test 2: English written word to Japanese spoken sound (Reading 2: English to Japanese). This test was another new test because students weren't trained the relationship between English written word and Japanese spoken sound. In this post test, an English written word was presented as a sample stimulus and the students were required to translate it into Japanese by reading aloud.

Figure 3 shows the relationship between the sample stimuli and required response for each of the post tests. In Figure 3, the black line labeled "Writing 1" represents Writing test 1: picture to English written word. The black dotted lines labeled "Writing 2" represent Writing test 2: Japanese spoken sound to English written word, "Reading 1" represents Reading test 1: English written word to English spoken sound, and "Reading 2" represents Reading test 2: English written word to Japanese spoken sound. Gray lines represent the stimulus relationships between the picture, Japanese word, and Japanese sound, which the participants have already acquired. The students could read, write and comprehend Japanese correctly, and they could also read and write English letters correctly. In the present study, only the stimulus relationship between pictures and English written words was trained. Figure 3 shows that pictures as sample stimuli were presented on the computer in the Writing 1: picture to English. Japanese spoken sounds were presented verbally by the experimenter in the Writing 2: Japanese to English. English written words were presented on the computer in Reading 1: English to English and Reading 2: English to Japanese. Writing 2: English to Japanese and Reading 2: English to Japanese were conducted to evaluate the effect of transfer from two types of training. 


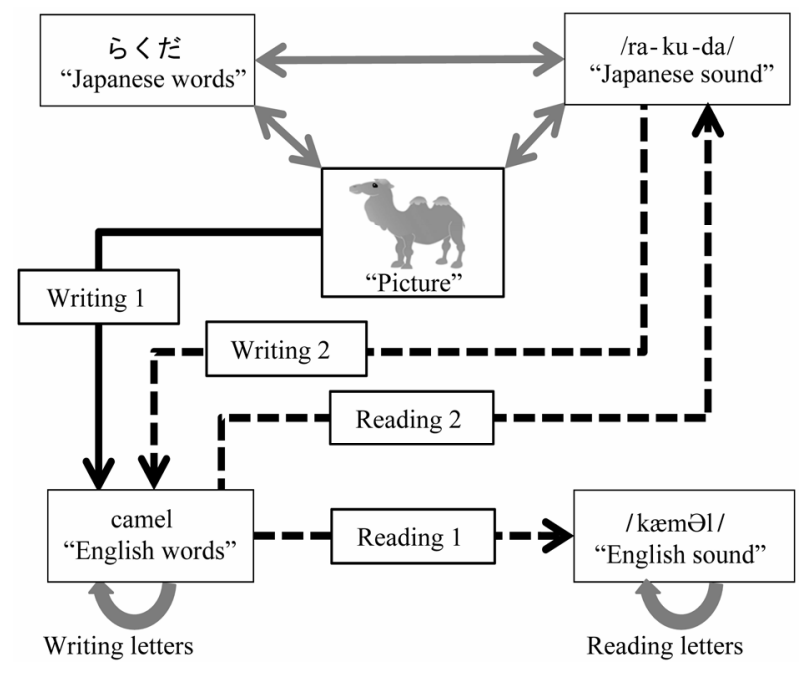

Figure 3.

Relationship between sample stimuli and required response in each of post tests. Black line labeled "Writing 1" represents Writing test 1: picture to English written word. Black dotted lines labeled "Writing 2" represent Writing test 2: Japanese spoken sound to English written word, "Reading 1" represents Reading test 1: English written word to English spoken sound, and "Reading 2" represents Reading test 2: English written word to Japanese spoken sound. Gray lines represent stimulus relationships, which the participants have already acquired.

\section{Data Analysis}

Because the all of stimuli the students learned were unknown at the time of pre-test and none of them could write down or read the word correctly. Therefore, we only analyzed the result from post test. The binominal test was used to compare the performance in the MTS and CRMTS procedures. For the statistical analysis, we calculated percent correct for the four students together. In each post test, each student received six word-length categories ( 3 to 8 letters); thus there were 24 samples to analyze for each post test. Each student experienced two types of training procedure. Therefore, the effect of training procedure was compared across all students.

To determine which percent correct was used for this binominal test, we compared the percent correct of writing and reading based on the four post tests for both procedures. Within a word-length category, we compared the percent correct for the MTS and CRMTS procedures and then determined which procedure showed a better result in this category. If the percent correct for MTS and CRMTS were same, the data of that category was not used for this analysis. After this determination, we counted the number of the higher percent correct categories for the MTS and CRMTS procedures. The number of the higher percent correct categories was counted for each student and then added across the four students based on the four post tests. For each post test, we analyzed the number of the higher percent correct categories of each MTS and CRMTS procedure.

While previous research (Stromer, et al., 1996; Stromer et al., 1998; Vedora \& Stromer, 2007) showed the effect of MTS and CRMTS procedures to transfer to writing skills for less than 5-letter-words, no studies reported that the acquisition of writing down more than 5-letter-words through the MTS and the CRMTS procedures. Therefore, in this analysis, we created three categories based on word-length categories. The results from word lengths with 3 to 5 letters were calculated first, those with 6 to 8 letters and all word length categories. Finally, the results from all word-length categories were calculated. An alpha level of .01 was used for all statistical tests.

For the individual analyses, percent correct was calculated based on the number of correct responses made out of 8 trials for each block.

\section{Reliability}

Due to the characteristics of two types of reading tests, two independent observers, including the experimenter, evaluated whether or not a correct response was made. Both listened as the students spoke and independently evaluated whether or not the response was correct. The observers evaluated all trials for each participant.

The reading response was recorded on the voice recorder and evaluated later by the one of two observers. Trial-by-trial interbserver agreement (IOA), calculated as the number of consistent scores, was used to determine interrater reliability. All of the reading tests in pre- and post tests were evaluated and calculated. The IOA values were $94 \%$ for the pre-test for Reading 1: English to English and $100 \%$ for the post test for the Reading 1: English to English and $100 \%$ for the post test for the Reading 2: English to Japanese. These values indicate satisfactory agreement. Ratings were made from the voice recorded spoken responses. Kappa (Cohen, 1968) was calculated to measure interrater reliability for the vocal responses, and was found to be high for ratings of responses by Tomo (.93), Ryo (.96), Atsushi (.91), and Sho (.93).

\section{Results}

\section{Statistical Analysis}

Scores of the binominal test in the Writing 1: picture to English were calculated. Although the effect of the CRMTS procedure was not statistically significant compared with the MTS procedure in the results for word-length category 3 to $5, \mathrm{n}=5$, $\mathrm{P}(4 \leq \mathrm{x} \leq 5)=0.38$, it was statistically significant for word-length category 6 to $8, \mathrm{n}=12, \mathrm{P}(\mathrm{x} \leq 12)=0.00$, and in all of word-length categories, $\mathrm{n}=17, \mathrm{P}(16 \leq \mathrm{x} \leq 17)=0.00$. In the Writing 2: Japanese to English, the effect of the CRMTS procedure was not statistically significant compared with the MTS procedure for word-length category 3 to $5, \mathrm{n}=5$,

$\mathrm{P}(4 \leq \mathrm{x} \leq 5)=0.38$. However, it was statistically significant for word-length category 6 to $8, \mathrm{n}=12, \mathrm{P}(\mathrm{x} \leq 12)=0.00$, and in all of word-length categories, $\mathrm{n}=17, \mathrm{P}(16 \leq \mathrm{x} \leq 17)=0.00$. In the Reading 1: English to English, in which the sample stimuli were English written words that were to be read in English, the effect of the CRMTS procedure was not statistically significant compared with the MTS procedure for word-length category 3 to $5, \mathrm{n}=4, \mathrm{P}(\mathrm{x} \leq 0)=0.12$, for word-length category 6 to $8, \mathrm{n}=$ $5 \mathrm{P}(4 \leq \mathrm{x} \leq 5)=0.38$, or for all of word-length categories, $\mathrm{n}=9$, $\mathrm{P}(0 \leq \mathrm{x} \leq 4)=0.50$. In the Reading 2: English to Japanese, the effect of the CRMTS procedure was not statistically significant compared with the MTS procedure for word-length category 3 to $5, \mathrm{n}=2, \mathrm{P}(\mathrm{x} \leq 2)=0.25$, for word-length category 6 to $8, \mathrm{n}=$ $6, \mathrm{P}(4 \leq \mathrm{x} \leq 6)=0.34$, or for all of word-length categories, $\mathrm{n}=$ $8, \mathrm{P}(4 \leq \mathrm{x} \leq 8)=0.64$.

\section{Data Analysis}

For the all participants, Tomo took an average of 5.50 blocks, Ryo took 5.33 blocks, Atsushi took 5.67 blocks, and Sho took 6.17 blocks to complete two types of trainings. Figure 4 showed the mean percentages of correct response for four types 

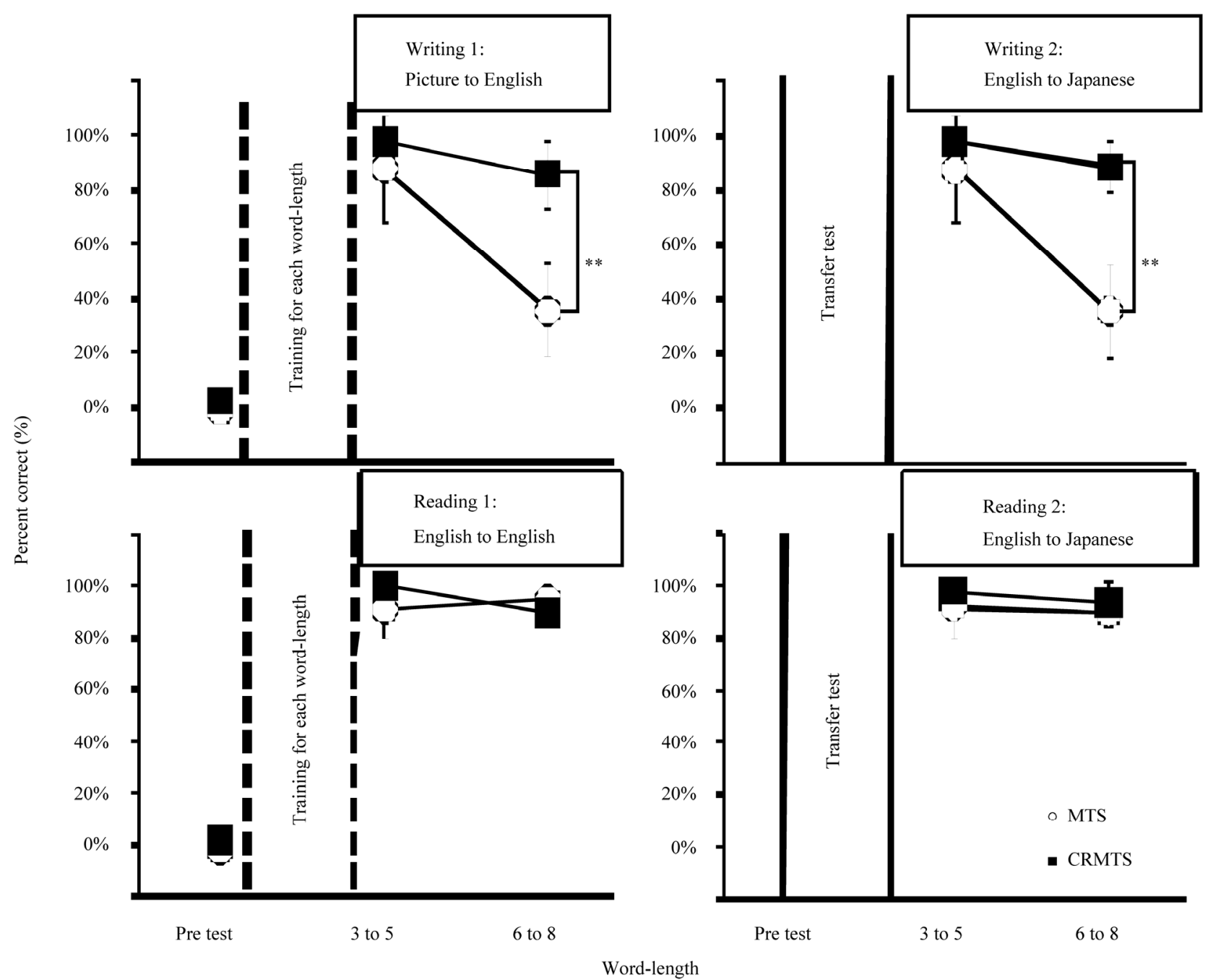

Figure 4.

Mean percentages of correct responses for four types of post test, the Writing 1: picture to English (top left), Writing 2: Japanese to English (top right), Reading 1: English to English (bottom left), and Reading 2: English to Japanese (bottom right). ${ }^{* *} p<.01$.

of post test in 3, 4, 5 word-length categories and 6, 7, 8 wordength categories. Figure 4 shows mean percentages of correct responses for four types of post test, the Writing 1: picture to English (top left), Writing 2: Japanese to English (top right), Reading 1: English to English (bottom left), and Reading 2: English to Japanese (bottom right). For both writing tests, the effects of training procedure differed in the two word-length categories (3-5 versus 6-8 letters). For shorter words (3-5 letters), the MTS and CRMTS procedures were similarly effective: $88 \%$ and $98 \%$ correct on both writing tests, respectively. For longer words (6 - 8 letters), however, the MTS procedure produced lower correct percentages than the CRMTS procedure: $35 \%$ versus $85 \%$, respectively, on Writing 1: picture to English and 35\% versus 89\%, respectively, on Writing 2: Japanese to English.

On the other hand, for both reading tests, the MTS and CRMTS procedures produced similar results in the two wordength categories. For shorter words ( 3 - 5 letters), the MTS and CRMTS procedures were both effective, producing $91 \%$ and $100 \%$ correct, respectively, on the Reading 1: English to English and $92 \%$ and $98 \%$, respectively, on the Reading 2: English to Japanese. For longer words ( 6 - 8 letters), percentages correct for the MTS and CRMTS procedures were very similar: 95\% versus $90 \%$, respectively, on the Reading 1: English to English and $90 \%$ versus $94 \%$, respectively, on the Reading 2: English to Japanese.

\section{Discussion}

The present study compared the effects of MTS and CRMTS on transfer to hand writing for ESL students with developmental disabilities. All of the students were able to acquire English writing skills through both MTS and CRMTS procedures. The students were able to match the sample stimuli with comparison stimuli by choosing words or by typing words on the computer. Even though they were not given any kind of training in writing, they also showed transfer to hand writing. The results of the present study therefore replicated those of previous research (Stromer et al., 1996; Vedora \& Stromer, 2007) in which transfer to a writing response via MTS and CRMTS procedures was reported.

We newly found training effects to be different for words of different lengths, with the difference occurring between words of 5 and 6 letters. In the 3 to 5 letter-word-length categories, 
results in writing after two types of trainings were similar to each other and did not show a statistically significant difference (Stromer, et al., 1996; Stromer et al., 1998; Vedora \& Stromer, 2007). On the other hand, for words 6 or more letters in length (the 6 to 8 letter word-length categories), all students showed better results in acquiring English writing skills after the CRMTS procedure. This result suggests that the CRMTS procedure was more effective for writing transfer in longer words compared to the MTS procedure. For shorter words, the MTS procedure was more useful and easier for students to use to acquire English writing skills because this training procedure took a much shorter time to complete than the CRMTS procedure.

Based on the present study, we suggest that the cut-off point to switch the training procedure is the word-length category between 5 and 6 letters as can be seen in the top two charts of the Figures 4. The results of Tomo and Atsushi especially reflect our suggested cut-off point. Tomo and Atsushi scored $100 \%$ correct response on the two types of writing tests after both MTS and CRMTS training in 5 letter-words category. However, in 6-letter-words category, while Tomo only scored $25 \%$ and Atsushi had $40 \%$ correct response on both writing tests after MTS training, they scored respectively $100 \%$ after CRMTS training. Thus, students who take about 5.50 blocks to finish the training might be expected to show this tendency.

For students who can reach the criterion faster, such as Ryo, there may be little difference between the results of the two procedures across all word-length categories. He scored 58\% correct response on both writing test, Writing 1: picture to English and Writing 2: Japanese to English, while others scored about $30 \%$ in the word length 6 to 8 letters categories. This type of participant may be able to construct stimulus relationships between pictures and English written words easily, so that they can acquire and transfer English writing skills even after training using the MTS procedure with longer words.

Although the MTS procedure seemed effective in the acquisition of English writing skills for short words, Sho demonstrated less effectiveness of the MTS procedure. Sho was actually able to write down the correct words with correct writing. However, he took more than six blocks to complete his training, suggesting that he had difficulty in acquiring the stimulus relationships between pictures and English written words. For this type of student, the CRMTS procedure may be better even for shorter word.

The present study also extended the previous results by demonstrating transfer of writing for untrained stimulus relationships. Our students were trained on only one stimulus relationship, which was between a picture and an English written word, in two types of trainings. However, they were able to acquire four stimulus relationships. One of them was trained stimulus relationship between a picture and an English written word, and three of which, Japanese spoken sound to English written word, English written word to Japanese spoken sound, and English written word to Japanese spoken sound, were acquired without training. As suggested in Figure 3, the relationship between an English written word and an English spoken sound, which was not actually trained, was established because differential feedback procedure constructed equivalent relationship during training (Dube \& McIlvane, 1995; Yamamoto \& Shimizu, 2001; Sugasawara \& Yamamoto, 2007).

As seen in the bottom two charts of the Figure 4, all of the students showed the acquisition of English reading with differential feedback. This result was suggested in previous studies (Vedora \& Stromer, 2007). Based on these results, we conclude that English spoken sound stimuli presented as differential feedback constructed stimulus relationship with pictures and written letters, and construct equivalent relationship. And learning to choose the words or type the words on the computer with differential feedback was effective for the acquisition and the improvement of reading skills even in ESL students with developmental disabilities.

In the present study, we extended some of the findings of Vedora and Stromer (2007). In their study, they focused on the acquisition of writing of the names of words. In the present study, we didn't focus only on the acquisition of words' name writing but also on Japanese dictation translating, English reading, and English translation reading. Figure 4 suggests that all students successfully acquired English writing skills and reading skills. The paradigm of equivalent relations can be extended to apply ESL students with developmental disabilities.

The present study has several limitations. One limitation was the small size of the sample. We only had four students with ASD, and a larger number of participants will provide more comprehensive data in the future research. Another limitation was that the length of the exposure of the sample stimulus on one trial was quite different in the two procedures. In the MTS procedure, the sample stimulus was exposed for about two seconds on each trial, because the participant only had to click the correct word. However, in the CRMTS procedure, the stimulus exposure was about ten seconds on each trial, because the participant had to type the word. It can be argued that the difference in the exposure times could have affected the results. Therefore, future research should consider controlling the sample stimuli according to not only the number of presentations but also the length of time they are presented.

\section{Acknowledgements}

This work has been supported by Global Center of Excellence (GCOE) program and Japan Society for the Promotion of Science (JSPS). This work was permitted by Keio University Institutional Review Board (IRB) in Faculty of Letter.

\section{References}

Cohen, J. (1968). Weighted kappa: Nominal scale agreement with provisions for scale disagreement or partial credit. Psychological Bulletin, 70, 313-320, doi:10.1037/h0026256

de Rose, J. C., de Souza, D. G., \& Hanna, E. S. (1996). Teaching reading and writing: Exclusion and stimulus equivalence. Journal of Applied Behavior Analysis, 29, 451-469. doi:10.1901/jaba.1996.29-451

Dube,W. V., \& McIlvane,W. J. (1995). Stimulus-reinforcer relations and emergent matching to sample. The Psychological Record, 45, 591-612.

Erhardt, R. P. (1988). Developmental hand dysfunction: Theory assessment treatment, Austin: Pro-Ed.

Hauck, J.A. \& Dewey, D. (2001). Hand preference and motor functioning in children with autism. Journal of Autism and Developmental Disorders, 31, 265-277. doi:10.1023/A:1010791118978

Layng, T. V. J., Twyman, J., \& Stikeleather, G. (2004). Engineering discovery learning: The contingency adduction of some precursors of textual responding in a beginning reading program. The Analysis of Verbal Behavior, 20, 99-109.

Ministry of Education, Culture, Sports, Science and Technology. (2002). Developing a strategic plan to cultivate "Japanese with English Abilities". Tokyo: Ministry of Education, Culture, Sports, Science and Technology.

Ohba, S. (1996). Problems and improvement of teaching handwriting in a regular class to children with writing difficulties. The Japanese 
Journal of Special Education, 33, 15-24.

Sidman, M. (2000). Equivalence relations and the reinforcement contingency. Journal of the Experimental Analysis of Behavior, 74, 127146. doi:10.1901/jeab.2000.74-127

Stromer, R., Mackay, H. A., Howell, S. R., McVay, A. A., \& Flusser, D. (1996). Teaching computer-based writing to individuals with developmental and hearing disabilities: Transfer of stimulus control to writing tasks. Journal of Applied Behavior Analysis, 29, 25-42. doi:10.1901/jaba.1996.29-25

Stromer, R., Mackay, H. A., McVay, A. A., \& Fowler, T. (1998). Written lists as mediating stimuli in the matching-to-sample performances of individuals with mental retardation. Journal of Applied Behavior Analysis, 31, 1-19. doi:10.1901/jaba.1998.31-1

Sugasawara, H., \& Yamamoto, J. (2007). Computer-based teaching of word construction and reading in two students with developmental disabilities. Behavioral Intervention, 22, 263-277. doi:10.1002/bin.248
Sugasawara, H., \& Yamamoto, J. (2009). Computer-based teaching of Kanji construction and writing in a student with developmental disabilities. Behavioral Intervention, 24, 43-53. doi:10.1002/bin.271

Vedora. J., \& Stromer, R. (2007). Computer-based writing instruction for students with developmental disabilities. Research in Developmental Disabilities, 28, 489-505. doi:10.1016/j.ridd.2006.06.006

Wechsler, D. (1998). Wechsler intelligence scales for Children (3rd ed.). San Antonio: The Psychological Corporation.

Yamamoto, J., \& Miya, T. (1999). Acquisition and transfer of sentence construction in autistic students: Analysis of computer-based teaching. Research in Developmental Disabilities, 20, 355-377. doi:10.1016/S0891-4222(99)00017-7

Yamamoto, J., \& Shimizu, H. (2001). Acquisition and expansion of Kanji vocabulary through computer-based teaching in a student with mental retardation: Analysis by equivalence relations. Japanese Journal of Special Education, 38, 17-31. 